\title{
Types of creativity
}

\author{
Arne Dietrich ${ }^{1}$ \\ Published online: 20 August 2018 \\ (C) Psychonomic Society, Inc. 2018
}

\begin{abstract}
Imagine the impact if we were to understand, and thus could reliably enhance, something - anything - about how creativity works in the brain. The fact that this prospect is not anywhere in sight makes it clear that no reliable progress has been made on the mechanisms underlying creativity over the last half century. Indeed, with the divergent thinking paradigm shown to be theoretically incoherent for neuroscience, there currently is no viable experimental approach to tackle the problem. Given that creativity is a complex and multifaceted concept, the obvious way forward is to parse it into subtypes. This paper presents a theoretical framework that divides the concept of creativity into three distinct types: a deliberate mode, a spontaneous mode, and a flow mode. Unlike previous attempts, the three creativity types are explicitly defined and delineated from one another based on established concepts in cognitive psychology and neuroscience. Moreover, to maximize the framework's heuristic value, this is done separately at three different levels of description: (A) neuroanatomy, (B) processes, and (C) evolutionary algorithms (EAs), or, more precisely, different parameters of EAs. This new theoretical framework advances the field in two significant ways. First, by defining the subtypes in terms of concepts that exist in mainstream psychology and neuroscience, they are valid subtypes, as they can be theoretically defended. Second, by providing a solid theoretical rationale to investigate a more circumscribed aspect of the larger problem, the framework provides a more targeted, and realistic, line of attack that will eventually lead to more meaningful data about the neural mechanisms of creativity.
\end{abstract}

Keywords Evolutionary algorithms $\cdot$ Flow $\cdot$ Prediction $\cdot$ Prefrontal cortex $\cdot$ Sightedness

\section{Introduction}

Creativity is the fountainhead of our civilizations and a defining characteristic of what makes us human. But for all its prominence at the apex of human mental faculties, we know next to nothing about the cognitive and neural mechanisms that generate creative ideas (Dietrich, 2015). Careful reviews of the literature have shown that the field is heavily fragmented and theoretically incoherent (Abraham, 2013; Dietrich \& Kanso, 2010; Sawyer, 2011; Weisberg, 2013; Yoruk \& Runco, 2014) (for opposing views, see Beaty, Benedek, Silvia, \& Schacter, 2016; Fink \& Benedek, 2014). In stark contrast to other domains of psychology and neuroscience, we do not have, at this point, a single cognitive or

Arne Dietrich

arne.dietrich@aub.edu.lb

1 Department of Psychology, American University of Beirut, Beirut 1107-2020, Lebanon neural mechanism to explain the extraordinary creative capacities of an Einstein or a Shakespeare.

It is uniformly agreed upon that creativity is a complex and multifaceted construct that can manifest itself in a myriad of different ways (Abraham, 2016; Dietrich, 2004a; Smith, Ward, \& Finke, 1995). For this reason, it is also uniformly agreed upon that creativity must be subdivided into different types if it is to be made tractable, especially for research in cognitive neuroscience. However, serious and solid theoretical work on developing suitable subtypes is lacking.

Historically, several attempts have been made to decompose creativity into subtypes. The most influential has been the concept of divergent thinking, defined as the ability to generate multiple solutions to an open-ended problem (Guilford, 1950, 1967). With the subsequent development of standardized psychometric instruments (Mednick, 1962; Torrance, 1974), the concept of divergent thinking has become the standard experimental paradigm for creativity.

The logical and methodological contradictions inherent in divergent thinking are now well documented (Abraham, 2018; Dietrich, 2015; Ward et al., 1999; Weisberg, 2013). Most importantly, there are two main problems that render 
it, as is, unusable for neuroscience research. First, divergent thinking is a false category formation (Dietrich, 2007a, 2015). Since the exact opposite - convergent thinking - can also produce creative ideas (Runco, 2004; Simonton, 2015), the obvious question arises of what, exactly, is creative about divergent thinking? If both divergent and convergent thinking can lead to both creative and non-creative thinking, divergent thinking is incapable of identifying the processes that turn normal thinking into creative thinking. The treatment and the control condition cannot contain the same variable.

Second, divergent thinking is still a compound construct consisting of many different and separate mental processes (Ward et al., 1999). It therefore does nothing to address the initial problem, the fallacy of taking creativity as a monolithic entity (Dietrich \& Haider, 2015). Although this, too, is widely acknowledged, it has not led to a research program of further dividing divergent thinking into subtypes that are perhaps more tractable by neuroimaging tools. In fact, the concept of divergent thinking has not undergone any further theoretical refinements in that direction and is used today the way it was initially conceived of over half a century ago.

For neuroimaging, both theoretical confounds - false category formation and compound construct - combine to make defeat certain. Simply put, if you fail to isolate the subject matter of interest in your study, you cannot use neuroimaging to hunt for mechanisms. You just don't know what the brain image shows!

There are additional problems with divergent thinking, such as the low ecological validity of the psychometric tests (Kerr \& Gagliardi, 2003), the fact that tests of divergent thinking are not tests of creativity - a point made by the custodians of the Torrance Test themselves (Runco, 2004; Cramond \& Kim, 2009) - and the widespread practice of overselling the findings from this divergent-thinking paradigm as discoveries about the whole of creativity, in the scientific literature and in the media (Dietrich, 2015).

A still bigger problem for the paradigm, however, is that divergent thinking seems to be a dead end. First, the concept cannot be linked to the knowledge base of mainstream psychology and cognitive neuroscience, as no other area of the behavioral and brain sciences makes use of it. Second, and more importantly, there is no effort underway, theoretically or experimentally, to break divergent thinking down further so as to link it to the kinds of mental processes we use to operationalize all other psychological phenomena, such as working memory, cognitive control, perceptual processes, semantic memory, inhibition, executive attention, or processes of social cognition. Knowledge of which individual mental processes, and to what extent, go into divergent thinking could potentially attenuate the problems of the false category formation and compound construct and perhaps make aspects of divergent thinking tractable with brain imaging tools in the future. But the divergent thinking paradigm taps into psychological and neuroscientific concepts only for the interpretation of the findings - default mode network (DMN), for instance (Beaty et al., 2016; Fink \& Benedek, 2014). What is needed, however, is to understand the concept of divergent thinking itself in terms of these individual processes so that the experimental methodology producing the findings in the first place is conceptually grounded.

There have been other proposals to partition, or better delineate, creativity over the years, be it in terms of processes (defocused attention, latent inhibition, remote associations, lateral thinking, low arousal, intelligence, alpha synchrony, etc.), states of consciousness (madness, daydreaming, REM sleep, flow, mindfulness, unconscious thinking, etc.), or neuroanatomy (right brains, prefrontal cortex, default mode network, network connectivity, etc.) (Baird et al., 2012; Beaty et al., 2016; Boden, 1998; Carson, Peterson, \& Higgins, 2003; Chrysikou, 2018; DeBono, 1968; Dijksterhuis, 2006; Fink \& Benedek, 2014; Jamison, 1993; Jung, Mead, Carrasco, \& Flores 2013; Kounios \& Beeman, 2014; Martindale, 1995, 1999; Mednick, 1962; Nikolaidis \& Barbey, 2018; Pfenninger \& Shubik, 2001; Schooler \& Melcher, 1995; Singer, 1975; Wagner, Gais, Haider, Verleger, \& Born, 2004), but they have all suffered from the same basic errors: false category formation or compound construct, or both. If the recent neuroscience research on creativity has revealed anything, it is the need for the theoretical development of valid subtypes of creativity that carves nature at the right joints.

A further complication for neuroscience research on creativity is the Vaudeville conception, the position that creativity might be fully distributed and embedded in the brain (Dietrich, 2004a). The notion follows, as a matter of consequence, from two basic concepts in neuroscience, nonlinearity and modularity (for further explanation, see Dietrich, 2015), and it takes seriously the view that creativity is a highly complex and multidimensional phenomenon. The Vaudeville conception holds that neural circuits that handle specific information to yield non-creative combinations of that information normal thinking, if you like - must be the same neural circuits that generate creative combinations of that information. Given that mathematics, car-parking, and painting engage totally different brain networks, so must creative mathematics, creative car-parking, and creative painting. What neuroimaging studies of creativity find, then, would entirely depend on how we decide to look, a logic that is supported by the highly variegated results of the field (Dietrich \& Kanso, 2010; Sawyer, 2011; Yoruk \& Runco, 2014). We might think of creativity as a cohesive entity in psychological terms, a character trait that some people have more than others - notice the singular - but creativity, as such, might not exist as a distinct and separate entity in the brain. The whole rationale of neuroimaging studies rests on the assumption that creativity, or divergent thinking, is a discrete thing in the brain and that that thing is detectable by 
neuroimaging tools. The tacit assumption here is that there is such a thing as 'normal' thinking to which an extra something the creative bit - is specifically added to make the sparkling difference. Few people would probably subscribe to this position once it is laid bare, but without it neuroimaging makes little sense. And it would not matter if the creative bit is a place(s), a network(s), or is added by enhanced connectivity between networks (for additional details on network connectivity, see Beaty et al., 2016); the tacit assumption is the same. In that way, creativity might resemble consciousness, which also is not tackled as a monolithic concept using neuroimaging, for the same distributed and embedded - reasons.

In contrast to other areas of creativity research (Runco, 2004), the neuroscientific study of creativity is adrift from the rest of the psychological sciences and finds itself in a theoretical arid zone that has perhaps no equal in psychology. The lack of a clear and detailed conceptual understanding of the construct of creativity leaves the field with no viable empirical paradigm to study its cognitive and neural mechanisms. It is the aim of this paper to sketch the outline of a new approach that can make the first cautious steps towards amending this situation.

\section{Rationale for the types}

In light of the complex and multifaceted nature of creativity, the obvious starting point is to parse creativity into different types. To avoid the pitfalls of earlier proposals attempting to do this - false category formation and compound construct the present theoretical framework takes a new line of attack. To enhance the prospect that the derived subtypes are indeed valid subtypes of creativity, they are explicitly defined in terms of mainstream behavioral, cognitive, neural, and evolutionary concepts. In other words, the subtypes are delineated from one another based on the same mechanisms we use to conceptualize all other mental phenomena.

One must be realistic about such an undertaking. Replacing divergent thinking with another simplistic proposal that associates the complex mental faculty of creativity with a single notion or dimension - say, cognitive dissonance or activity in the mirror neuron system - might come, due to its one-dimensionality, with an instantaneously testable experimental hypothesis. But this is neither likely to lead to meaningful data in the case of creative thinking nor is this a reasonable expectation, given how multifaceted the topic is and how far we are away from understanding the critical elements of its mechanisms. In consequence, the emphasis of the present theoretical framework is on making only those steps that can be anchored and thus theoretically defended. In trying to make some progress on consciousness, a topic that is similarly elusive, complex, and in its initial stages, Crick and Koch (2003) defined a framework as:
A framework is not a detailed hypothesis or a set of hypotheses; rather, it is a suggested point of view for an attack on a scientific problem, often suggesting testable hypothesis. ... A good framework is one that sounds reasonably plausible relative to available scientific data and turns out to be largely correct. It is unlikely to be correct in all details. ... [It] acts as a guide (p.119).

Three different types of creativity are proposed. Each can be clearly circumscribed in terms of three different levels of description: (A) neuroanatomy, (B) processes (cognitive, computational, and physiological), and (C) evolutionary algorithms (EAs), or rather different parameters of EAs. While (A) and $(\mathrm{B})$ are proximate explanations, $(\mathrm{C})$ is an ultimate explanation (Mayr, 1981).

To start with a psychological account, creative products can come into existence by way of three distinct modes of information-processing (Dietrich, 2004a, 2004b). In the deliberate mode (DM), creative ideas are generated by deliberate iterations of trial and error. In the spontaneous mode (SM), creative ideas arise suddenly, spontaneously, and unintentionally in the mind. In the flow mode (FM), creative behavior comes about through fluid and effortless motion that bypasses consciousness altogether. The DM and SM modes are related to the distinction between analytical search strategy versus insight problem solving, which has existed in psychology for some time (Simonton, 1975). But this insight-analytic distinction has never been tied to cognitive or neural mechanisms beyond attentional processes (Zabelina, 2018) and the rightbrain theory (Kounios \& Beeman, 2014), or integrated with the FM to yield a more comprehensive picture of all the different ways humans can be creative. It should be stressed from the outset that a given creative act is not suggested to be the manifestation of one of these types in pure form, just as any other complex behavior cannot be reduced to one type or process. As an example, a song might be written in any of these three modes or by switching back-and-forth between them. But each mode does have unique properties that bring different advantages and disadvantages.

Before we detail the underlying mechanisms involved, it might help to flesh this out further. The DM is associated with conscious processing, feelings of agency, executive or topdown attention, effort, volitional control, purposeful memory retrieval, intentionality, and planning. In romanticizing about the creative process, some might argue that this type of analytical thinking does not count as "real" creativity, but it is clear that we can also converge on creative solutions by logically, systematically, and consciously reasoning through a given problem space. What would we otherwise make of Edison's "empirical dragnet" method that yielded a total of 1,093 patents; Watson and Crick's disciplined approach of testing the stability of DNA base pairs; Bach's assembly-line tactic of composing hundreds of cantatas; the imaginative ways in 
which NASA engineers solved the problems of the otherwise doomed Apollo 13 mission; or the countless occasions we hone in on creative answers by methodically eliminating alternatives.

In contrast to this hard-thinking way of the DM, the SM is associated with the opposite set of attributes: unconscious processing, no sense of agency, inattention or bottom-up attention, no volitional control, effortlessness, undirected memory retrieval, and the eventual representation of the insight in working memory as surprising, intuitive, and accidental. From Kekulés daydream of whirling snakes forming a (benzene) ring, to Newton watching apples fall (a myth likely originating from Voltaire), Coleridge's conception of the poem Kublai Khan, and Archimedes displacing bathwater, such flashes of insight are the very cliché of the creative genius.

The FM is a third way to be creative. It can be identified as a distinct type on the basis of one defining characteristic the other two do not require: physical motion. This has far-reaching consequences for the kind of creative product that can be made, the creator's phenomenology, and the mechanisms underlying it. Flow is a highly attentive state of consciousness characterized by effortless, fluid, and graceful motion (Csikszentmihalyi, 1996). A flow experience ensues when one becomes so deeply focused on a task and pursues it with such passion that all else disappears, including the passage of time, worry of failure, selfreflection, any meta-analytical processes, or the sense of authorship. The person enters a tunnel, an almost euphoric state of bliss, in which the task at hand is performed, without strain or effort, to the best of the person's ability (Dietrich 2007b). According to Csikszentmihalyi (1996), any activity can produce flow as long as it is a challenging task that demands intense concentration and commitment, contains clear goals, provides immediate feedback, and is perfectly matched to the person's skill level. As such, it is not limited to athletes or performing artists, but can also occur in more unsung activities. The importance of a skilled movement sequence as a defining feature of FM cannot be overstated. Being totally immersed in something - watching a mesmerizing movie, reading a gripping novel, deep meditation, etc. - does not qualify as a flow state. The essence of flow is the merging of perception and action, the smooth, rapid-fire integration of sensory input and motor output that cleanly bypasses higher-order thought and consciousness.

This rough and initial sketch makes clear already that wholesale statements about creativity being linked to any one thing, process, place, or feature are hollow and cannot hold water. Since the ways in which creativity works can be utterly different, and at times even opposing, creativity, as a whole, cannot possess such a one-dimensional cognitive or neural signature. This applies to the compound construct of divergent thinking as well. In consequence, broad claims about the mechanisms of creativity per se must be regarded, as defined by Uttall (2001), as a form of phrenology. They are all false category formations and betray a tacit commitment to the monolithic entity fallacy.
Finally, it might be useful to disentangle a few common confusions that often surface when thinking about types of creativity. First, domains are not types. The former is about content, the latter is about mechanisms. For instance, in the same way reading attention is not a separate type of attention, artistic, spatial, or verbal creativity are not separate types of creativity. Basic neural and cognitive mechanisms must be, by and large, independent of content, although it is readily conceivable that we might discover exceptions to that in the future. Second, variation and selection are not different types of creativity (see, for instance, Jung et al., 2013); they are components of the creative process that are present in all types. In other words, all types of creativity can be described in terms of EAs, and an EA always contains both processes, variation and selection. Third, barring an exception that will be spelled out later on, EAs are not one type of creativity, as if there are other types of creativity that cannot be described in terms of EAs (Dietrich, 2015). As an ultimate explanation, EAs are a different level of description for all three types of creativity, and the proximate explanations of (A) neuroanatomy and (B) processes cannot be used in replacement of it.

\section{Three levels of mechanisms}

To maximize the heuristic value of the proposed theoretical framework, the three modes of creativity are tied to their underlying mechanisms at three different levels of description: (A) neuroanatomy, (B) processes, and (C) EAs. This is not intended to be a hierarchical setup. Rather, the descriptions are complementary, often expressing the same thing but at a different explanatory level and in a different language. This also does not produce redundancies, but is instead explicitly meant to facilitate the identification of as many points of contact between creativity and the various areas of the psychological sciences. Also, no claim is made that the underlying mechanisms described here represent an exhaustive list. But the present theoretical framework does focus heavily on those aspects that also dominate the mainstream literature.

For (A) neuroanatomy, differences in the three modes are specified for the following key aspects: prefrontal cortex activity, neural network configuration or task sets, and explicitimplicit systems (for an overview of task sets and related concepts, see Monsell, 2003 and for the explicit-implicit distinction, see Dienes \& Perner, 1999). For (B) processes, three sublevels - cognitive, computational, and physiological - could be considered, most of which change as a function of changes in (A) neuroanatomy. The focus here will be on cognitive processes, such as the various higher-cognitive functions - working memory, attention, cognitive flexibility, inhibitory control, memory retrieval, cognitive biases, functional fixedness, social norms, schemas, agency, etc. - spreading activation, task-set inertia, speed of processing, procedural memory, or processing 
efficiency. But, when applicable, computational processes, such as Bayesian inferences (for an introduction, see Wolpert, Doya, \& Kawato, 2003), or physiological processes, such as levels of arousal or temporal synchrony, will also be highlighted.

For (C) EAs, a number of additional complexities need to be drawn out before the differences among the three modes of creativity can be appreciated. For all the heat in the debate on cultural evolution, there is a remarkable, broad consensus on key issues. To a first approximation, the following is unanimously agreed upon: (1) Culture is an evolutionary system (Dawkins, 1976; Gould, 1979; Smith, 2013); (2) Culture is a system with a variational or variation-selection pattern of change (Lewontin, 1991; Richerson \& Boyd, 2005), and (3) Culture exhibits some coupling between variation and selection; that is, unlike biological EAs, the brain's EAs are not blind, but rather fall on a continuum of degrees of sightedness (Dietrich \& Haider, 2015; Kronfeldner, 2007; Simonton, 2013; for an introduction to sightedness, see Kronfeldner, 2010).

Recently, the brain's prediction system (for an overview, see Bar, 2009) has been proposed as the brain mechanism of the sightedness upgrade that cultural EAs possess (Dietrich, 2015). The ability to compute a goal representation that contains fitness values with which to predict the location of solutions with a probability greater than zero, even when the problem space is unknown, causes unique properties that change the way cultural EAs operate and the type of artifacts they can bring into existence. Most importantly, predictive computations yield degrees of sightedness, enable cognitive scaffolding and generate the feeling of foresight and intention of human creativity (for details, see Dietrich, 2015). The brain's predictive capabilities are known to differ for (1) explicit and implicit systems (Downing, 2009; Grush, 2004) and (2) engagement of higher-cognitive functions (Dietrich \& Haider, 2015; Moulton \& Kosslyn, 2009). In consequence, all three types of creativity can also be delineated on the basis of their EAs' sightedness parameter, which has implications for the search heuristics, scaffolding, and sense of foresight and agency of each type. Strictly speaking, predictive computations should fall under (B) processes, but they are better discussed under (C) EAs, as they are the reason for the differences in degrees of sightedness of each type.

\section{The deliberate mode}

Evidence from connectionist modeling, psychology, and neuroscience have converged on the view that conscious processing and other higher-cognitive functions involve competition between widely distributed representations that are strongly dependent on top-down prefrontal activity (Baars, 1988; Dehaene \& Changeux, 2011; Maia \& Cleeremans, 2005; Miller \& Cohen, 2001; Norman \& Shallice, 1986). Based on this literature, the DM is characterized by strong prefrontal cortex activation that biases and thus directs information processing (Dietrich, 2004a). It follows that the DM can be associated with the explicit system in terms of the dual-system (explicit-implicit) architecture (Dienes \& Perner, 1999; Haider \& Frensch, 2005; Reber, 1993) and, to a limited extent, with the central-executive network (CEN) in terms of the brain's large-scale networks (Bressler \& Menon, 2010; Raichle et al., 2001). Finally, in connectionist terms, the DM can be characterized as a strong task set, in which the node weights of the knowledge structure are set to highly polarized frequency of occurrence values (Dietrich \& Haider, 2017). Although task sets are standard explanations in cognitive psychology (Allport, Styles \& Hsieh, 1994; Dreisbach \& Haider, 2009; Monsell, 2003), the concept has not been applied to creative thinking, despite their obvious relevance to how we might conceptualize a given task, initially configure a task's potential solution spaces, or functional fixedness.

From the conclusions for (A) neuroanatomy, the following corollaries for (B) processes can be derived. In the deliberate mode, the explicit control and the prefrontal activation bring the toolbox of the higher-cognitive function to bear on the creative task, including working memory, executive attention, inhibitory control, conscious memory retrieval, and agency. It is for this intentional and effortful way that it is called the deliberate mode. One should not conclude from this, however, that creativity depends on all the computational power and cognitive flexibility these higher-order processes make possible. In an effort to provide a constant stream of reminders against false category formations, they also bring disadvantages (Dietrich, 2015). Top-down prefrontal activity is associated with the strong activation of social norms, schemas, and cognitive biases (Damasio, 1994; Miller \& Cohen, 2001), so that the effortful retrieval of knowledge from long-term memory and the recombinatorial shuffling of it in working memory is likely inherently biased towards general beliefs of what is considered true about the world. In other words, the search engine itself as well as the rearrangement of informational bits has built-in predispositions that are constrained by a number of parameters, such as biases, expectancies, preferences, schemas, experiences, or common sense. As such, the DM is more limited to creative insights that are more paradigmatic in type and rely on more close associations (Dietrich, 2004a).

Recasting the same tradeoff in network terminology, the DM's strong top-down input changes how spreading activation ripples through the knowledge structure. The frequency of occurrence values and the strength of their connections are recalibrated so that the task set now reflects the extent to which certain aspects of the task or a person's expertise and belief system are expected to contribute to a solution. These new calibrations then determine how information flows through that network. The more polarized the weight assignment, in either the plus or minus direction, the less likely it is for spreading activation to diffuse and establish remote associations. Such an opinionated knowledge structure is highly efficient - or highly 
inflexible, depending on the point of view - when it processes specific information (Dietrich \& Haider, 2017).

The DM's general pattern of activation also has consequences for the parameters of (C) EAs. The bringing online of the explicit system's full arsenal of higher-cognitive functions includes prediction processes as well (Dietrich, 2015; Downing, 2009). In the DM, the brain's neural simulators can thus compute highly informed goal representations that yield more degrees of sightedness when exploring unknown problem spaces. These superior EAs have strongly directional heuristics, the capacity to scaffold, and bring about the sense of foresight and agency (Dietrich, 2015). It is a powerful upgrade from the blind EAs of biological evolution and it can make short work of a given problem space. The tradeoff highlighted above can also be expressed at this evolutionary level of description. The advantage of advanced heuristics is efficiency (see Gigerenzer, \& Gaissmaier, 2011, for details). By pre-empting, a priori, counterintuitive paths or ideas orthogonal to accepted wisdom, a vast search space is trimmed down to a more manageable region that is more likely to contain a solution. This only works well, however, if the solution is indeed located in the predicted region of the problem space. To quip, while the DM has the advantage of limiting the solution space, it has the disadvantage of limiting the solution space!

In keeping with efforts to build bridges between different levels of description, we can ask what might constitute a selection process - an aspect of (C) EAs - in a connectionist network for (A) neuroanatomy and (B) processes. How could a fitness function be implemented in such a network? One possible mechanism is the speed of processing (Whittlesea, 2004). A weighted network creates processing speed differentials for different kinds of information that are run on it. In evolutionary language, a strongly polarized network corresponds to a case of strong selection pressure. Depending on the configuration, some information is processed more expediently than others. That information is simply a better fit for the preset processing pathways. The speed-of-processing parameter could serve as a selection process because it can discern value (Dietrich \& Haider, 2017). Individual differences in creativity can also be understood that way. A person with a different network setting, as determined by factors such as expertise or past experience, might process the same potential solution at a lower speed and would not select for it.

It should be evident at this point that the underlying mechanisms described here for the DM are incompatible with associating the whole of creativity with mind-wandering, right brains, defocused attention, remote associations, unconscious thinking, or the default mode network.

\section{The spontaneous mode}

For the SM, a very different picture emerges for all three levels of description. In removing the creative task from consciousness, there are significant changes for all aspects of (A) neuroanatomy. The SM has considerably weaker supervisory, top-down influences from the prefrontal cortex that guided the effortful thinking of the DM (Dietrich, 2004a). However, the SM must still be considered as part of the explicit system. It can draw on explicit long-term memory, use a scaffold - as evidenced by discontinuous insights - and, most importantly, the ongoing, unconscious processing has access to working memory as a creative idea. As we will see shortly, none of that is the case for processing in the encapsulated implicit system (Dienes \& Perner, 1999; Haider \& Frensch, 2005). Also, the SM could be loosely associated with the default mode network, but this association must be regarded as tentative at present due to the still limited understanding of the functional significance of that network. Finally, the SM could be characterized as a weak task set in which network nodes are reset to much less polarized frequency-ofoccurrence values (Dietrich \& Haider, 2017).

The adjustments are equally extensive for (B) processes. Once the mind is otherwise applied, the higher-cognitive functions go partially or fully offline. Processing for the creative task is now unconscious, lacks attentional control, and the highly selective retrieval of knowledge from long-term memory is as gone as is the sense of agency. The loss of all this computational power renders the SM a much less efficient information-processor. On the flipside, incubating the creative task also puts an end to the strong, top-down activation of schemas, expectations, and norms, and, with this prefrontal-imposed bias lifted, the SM has the potential to chance upon more paradigmshifting ideas or remote associations (Dietrich, 2004a).

Expressing the same tradeoff in connectionist language, as soon as the task set is demoted into the unconscious, the activation in this network changes in strength and quality. Right away, this triggers a drop off in overall network intensity, but the network continues to process the task due to task-set inertia (for an introduction to this concept, see Allport, Styles, \& Hsieh, 1994). With the loss of top-down influences from the prefrontal cortex, this general decline in activity is accompanied by the resetting of all relevant knowledge nodes to more moderate values. The task set representation of the creative task is now both weaker and more neutral. This has two effects for spreading activation. First, information processing is more diffuse, but this loss of sharpness and direction also comes with a wider reach in the network. Second, processing speed differentials are altered. The same information that might have run at low processing speeds in the DM's network configuration could do better in a more evenly organized network. Also, there is the possibility that speed of processing works as a strengthening mechanism that can determine which particular combination of information breaks through to working memory and becomes a creative insight (Dietrich \& Haider, 2017).

For (C) EAs, the SM must make due with greatly reduced prediction competencies (Dietrich, 2015). Without the benefit 
of higher-order, conscious thought, neural simulators are unlikely to have either full access to memory or the computational power to generate well-informed goal representations. The SM possesses, therefore, considerably less degrees of sightedness, its search heuristics are less directional, and the sense of foresight and agency can no longer be computed (Dietrich, 2015). The tradeoff here is the opposite from the one in the DM. Due to the reduced sightedness and inferior heuristics, the SM must contend with larger solution spaces and a more aimless search process, making it much less efficient. But if the solution is located outside the region of the solution space predicted by the DM's strongly directional heuristics, the EAs of the SM are also more likely to find it. To quip again, while the SM has the advantage of larger solution spaces, it has the disadvantage of larger solution spaces!

The SM should be expanded to accommodate the larger set of altered states of consciousness (ASC). Although the daydreaming described above is only a mild sort, it is generally considered an ASC along with a few other putative ASC, such as dreaming, meditation, hypnosis, flow, drug states, and the runner's high (Blackmore, 2005; Dietrich, 2007b). The transient hypofrontality theory (THT) has been proposed as a general brain mechanism that can account for a great number of phenomenological features common to all ASCs (Dietrich, 2003). It is based on the common conceptualization of brain areas and mental abilities into a functional hierarchy with the top layers in the prefrontal cortex contributing the most sophisticated elements of the conscious experience, such as selfreflection, working memory, executive attention, temporal integration, abstract thinking, cognitive control, volition, or the sense of agency. Analogous to peeling an onion, the THT simply postulates that alteration to consciousness involves the progressive downregulation of networks supporting the highest cognitive capacities, down the functional hierarchy, one phenomenological subtraction at a time, to those supporting more basic ones. Since all altered states share the

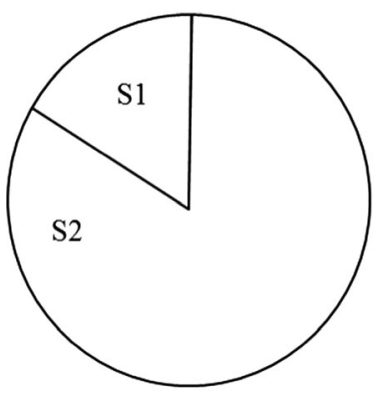

Fig. 1 The Efficiency-Unconventionality Tradeoff. No type of creativity is better per se. Which one is more suitable depends primarily on where in the problem space the solution is located, which, of course, we do not know ahead of time. A problem space can be conceptualized as a circle with the creator in the center. For the deliberate mode (DM; left circle), the high degree of sightedness of the problem space permits strongly directional search heuristics. Should the solution (S1) fall within the predicted region, the DM is a very efficient problem-solving mode that will gradual disappearance of mental faculties that depend on prefrontal input - with the exception of executive attention for some - ASCs include a transient state of hypoactivity, of various depths and extent for each altered state, in networks of the prefrontal cortex. As such, the consequences of the weakened, top-down prefrontal projections that apply to the creative thinking in the SM - little higher-cognitive functions, less sightedness, or more possibility for remote associations can be extended to ASC in general.

Clearly, neither type of creativity can be said to be better. Each has its advantages and disadvantages depending on the specific situation. The critical factor seems to be where in the problem space the solution is located, which, needless to say, we do not know ahead of time. Generally speaking, if it is in line with the current thinking, the DM is better; if it is orthogonal to it, the SM is better. Also, this analysis underscores the value of decomposing creativity into valid types when making claims about possible cognitive and neural mechanisms. While creativity, as a whole, cannot be associated with decreased prefrontal activity, default mode network, sleep, defocused attention, mind-wandering, unconscious thinking, or remote associations, a particular type of creativity - the SM - might be. This cannot be done with divergent thinking. Notice also that the DM and SM cannot be conflated with convergent and divergent thinking as both the DM and the SM contain both convergent and divergent thinking Fig. 1.

\section{The flow mode}

The FM is a radically different way of generating creative behavior. Unlike the DM and SM, both of which do not necessitate physical motion and their processing eventually ends with an active representation in working memory - the creative idea - the FM does require motion and it bypasses consciousness altogether. One feature of the FM - the need for motor efficiency - produces a cascading series of corollaries

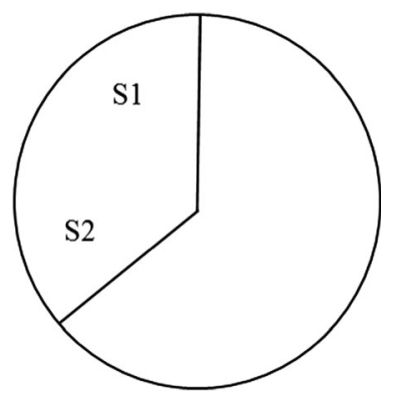

find the solution in short order. Should the solution (S2) be more unconventional and fall outside that region, the DM is not a good tool. The opposite holds for the SM (right circle). The SM cannot generate such strongly directional heuristics, resulting in larger solution spaces. With the loss of efficiency, the SM may not find either the conventional (S1) or the unconventional (S2) solution. But, unlike the DM, it has at least a chance to find the latter, because it was not excluded, a priori, from the pool of possibilities 
that fundamentally changes the FM's mechanisms at all three levels of description (Dietrich, 2015). For this discussion, it is more practical to combine the two levels of description of (A) neuroanatomy and (B) processes.

First, the FM is driven by the implicit system (Dietrich, 2004b). This follows, as a matter of consequence, from the flexibility-efficiency tradeoff between the explicit and implicit systems. The sophisticated and complex explicit system is based on higher-order mental representations and has evolved for cognitive flexibility. The simple and concrete-operational implicit system is based on a procedural representational format and has evolved for speedy and accurate motor execution (Dienes \& Perner, 1999; Haider \& Frensch, 2005; Reber, 1993). The key to understanding the flexibility-efficiency tradeoff is the computational fact that motor efficiency precludes cognitive flexibility and vice versa (Dietrich \& Audiffren, 2011; Dienes \& Perner, 1999). A system cannot be complex and fast at the same time, and the need for both at different times is likely the reason we have two distinct information-processing systems in the first place. This tradeoff must be rigorously applied to the FM, because the inherent processing efficiency of implicit knowledge is paramount to the fluid and effortlessness action of the FM.

Second, the FM requires the automatization of the motor skill (Dietrich, 2015). This is a direct corollary of implicit control. The implicit system is experience-based and can only develop a mental representation of the motor task by doing it. In other words, since the motor efficiency that defines the FM can only proceed from an implicit representation, the motor skill must be well practiced first. This is why novices learning a new skill do not report flow experiences (Csikszentmihalyi, 1996). In addition, given that the neural substrate of the implicit system is based on activity in the basal ganglia, cerebellum, and the supplementary motor area (Poldrack \& Packard, 2003), so must be the neural substrate of the FM. As such, the creativity that arises in the FM cannot be associated with either the CEN or the DMN.

Third, the FM requires a general state of transient hypofrontality (Dietrich, 2003, 2004b). This, too, follows from the need for motor efficiency. Explicit interference in the control of an automated motor skill - consciously thinking about the movements - decreases the quality of the movement (Beilock \& Carr, 2005; Ravizza, 1977), or, to state it the other way around, optimal performance of a real-time sensorimotor integration task is associated with maximal implicitness of its execution (Dietrich, 2004b). A graceful and fluid motor sequence cannot be micro-managed by the explicit system. Its representational format is abstract and higher-order and hence too slow to be applied to a specific situation in real time. A state of lower prefrontal activity, therefore, would minimize the chances that top-down, explicit processes compromise the smooth, implicitly-driven execution of a motor skill and ensure that a person can enter, or can stay, in flow. From an evolutionary perspective, a state of transient hypofrontality is beneficial in a pressure situation involving physical motion; it simply is not adaptive to engage higher-order analytical processes when the devil takes the hindmost (Dietrich \& Audiffren, 2011).

Moreover, the THT fits with FM phenomenology. While the movements themselves are performed with ease, the flow experience is marked by phenomenological subtractions of exactly those higher-cognitive functions that depend on prefrontal activity. With the exception of focused attention, the FM lacks conscious awareness, a sense of self and agency, intentionality, abstract analysis, mental time travel, or the ability to consider possible long-term consequences of the ongoing action. Should any of these complex mental faculties return, flow is gone (Dietrich, 2004b). Dennett (2004) once quoted the painter Philip Guston as saying:

When I first come into the studio to work, there is this noisy crowed which follows me there; it includes all of the important painters in history, all of my contemporaries, all the art critics, etc. As I become involved in the work, one by one, they all leave. If I'm lucky, every one of them will disappear. If I'm really lucky, I will too.”

It might be helpful at this point to draw out two issues that are potential sources of confusion regarding the mechanisms of the SM and FM. First, the two types of creativity should not be confused just because both are unconscious. As said, the SM emanates from the explicit system, as it can draw on explicit long-term memory, use a scaffold, and access working memory. The FM can do none of that. Knowledge in the encapsulated implicit system cannot become conscious through an internal, bottom-up process (Dienes \& Perner, 1999; Haider \& Frensch, 2005; Reber, 1993). It must go, as it does for the FM, through the circuitous route of involving actual behavioral performance (Dietrich, 2015). Put another way, the unconscious creativity in the SM is explicit; the unconscious creativity in the FM is implicit. Second, while both types cannot occur without the muffling of prefrontal-dependent, explicit processes, the effect of transient hypofrontality on the creative process differs for each type. In the SM, the prefrontal hypoactivity changes the way the explicit system itself processes information. In the FM, it facilitates the way the implicit system processes information. Also, since the task itself is automatized, the transient hypofrontality in the FM can also be much more profound because explicit supervision is not critical - and, indeed, is detrimental.

Before the differences that arise from (C) EAs are fleshed out, this can be summarized as follows: Creativity in the DM and SM are offline, outside real time, and tied to consciousness, while in the FM it is online, inside real time, and outside consciousness. Because of this, scientists rarely make use of the FM. A creative act in science typically does not require motor 
efficiency and can thus be done offline and outside real time. This does not hold for artists, as there are many forms of artistic creativity that require online performance and others that do not.

The shift from explicit to implicit control in the FM makes for a change in $(\mathrm{C})$ EAs that could not be any bigger. The reason for this are the fundamentally different prediction capacities of the implicit system (Dietrich, 2015; Downing, 2009; Grush, 2004). This is perhaps best illustrated by first distinguishing between known and unknown problem spaces (for an earlier discussion on problem spaces, see Boden, 1998).

The motor system does have a prediction mechanism. The system relies heavily on emulators and controllers, which are representations of the future (Wolpert, Ghahramani, \& Jordan, 1995). Indeed, it is likely in motor control where the capacity for predictive computation first evolved (Wolpert et al., 2003). However, the motor system can only run these predictions in a known problem space. Its concrete-operational setup cannot handle hypothetical situations, that is, predict the sensory consequences for a movement the body has never made (Dienes $\&$ Perner, 1999). The system simply lacks the computational means to generate controllers that are imaginary. Unlike the explicit system, then, it has no way to render a prediction in an unknown problem space. The fact that the motor system cannot compute an internal model of the entire sensorimotor arc is the reason why a first-time movement feels so weird (Frith, 1992). We simply cannot anticipate what we are going to see or feel. In sum, the motor system must already know the goal or movement end states, so that a predictor-controller pair can be established and allowed to converge using Bayesian inferences (Wolpert, et al., 1995). It should be obvious that a known problem space does not count as creativity; the problem space is already mapped out.

The execution of a skilled motor sequence - a known problem space for the motor system - is not even a variationselection EA. For all possible forward steps or predictors, there are principally known consequences or controllers. Instead, it is a mapping process in which no variationselection method is needed for the individual movement steps. With all predictor-controller pairs established, the algorithm that needs to be solved is essentially known. In evolutionary language, this can be described by a Lamarckian EA in which the forward steps are directed or instructed by their known adaptive values. The movement sequence can unfold fully sighted, directed by a whole host of acquired and internalized controllers. The equivalent in the explicit system of prediction processes in a known problem space would be, for instance, the game of chess or the planning out of one's morning chores. This is well-trotted territory, cases of strategic planning or effective decision-making in which the brain runs simulation chains that reason through a series of choice points in the future. In principle, there is (near total) sightedness of the adaptive landscape and the outcome of each step has (near total) predictability.
But creativity is an adventure into parts unknown, which, in motor control, only occurs if we try a totally new movement - snowboarding or dancing tango, for instance. In this case, the motor system has no way of making a prediction because it cannot compute a hypothetical controller. It does not know what the correct movement endpoints might be and without these controllers it would not even know the algorithm it needs to solve (Wolpert et al., 2003). The motor system must first acquire them and it can only accomplish that through action. Learning in the implicit system is stochastic and stepwise. The system tries out, by trial and error, solutions to environmental contingencies (Perruchet \& Vinter, 2002). Unlike the explicit system, which forms long-term memories that can then be fed into neural simulators, the implicit system embeds the new information into the network itself by slowly and gradually shifting the weights in its knowledge structure. These weight changes represent predictions that are then used the next time the situation arises. Motor learning is basically a process of acquiring controllers or reducing prediction errors (Wolpert et al., 2003).

In evolutionary terms, one could also say that learning is the process of gaining degrees of sightedness of the fitness landscape. The initial acquisition of a motor skill, then, is best described by a Neo-Darwinian EA. The lack of any kind of prediction competencies to simulate possible movement end states means that the motor system must approach unknown problem spaces blind. It has no controllers or sight of the fitness function. This also explains the disappearance of foresight and agency in the FM (Dietrich, 2015), as evidenced by the quote of Philip Guston, since these experiences also depend on prediction processes (Frith, 1992; Grush, 2004). The great difference to the DM and the SM, of course, is that the explicit system can compute goal representations in such unknown problem spaces. The difference in cognitive systems is also the reason why the efficiency-unconventionality tradeoff that exists between the DM and SM cannot be extended to the FM. The FM's implicit processing represents a non-linear break with the logic that less directional heuristics allow for more remote associations.

Incidentally, this is also why flow flows. This feeling of perpetual continuity is generated by three mechanisms. First, flow is a no-search, mapping process in which each step is automated and paired with a controller. Second, flow is a stepwise process since the implicit system cannot anticipate more than one step at the time. Without prediction, each step of the chain depends on the preceding one and triggers the next. This also makes it impossible for the FM to scaffold. Third, flow is free of meta-analytical, explicit interference that could disrupt the performance.

Given that the activity itself must occur in a known problem space, the question arises where in the FM is the creativity. In creating a painting, for instance, an artist's actual hand movements are nothing new to the motor system, and each stroke of 
the brush is mapped. Likewise, a jazz musician's technique must be skillful for improvisation to yield anything creative. Although there are differences between these two flow examples, as the former still allows for revisions while the latter does not, the creative act cannot lay in the steps themselves but must emerge from the overall pattern produced by the entire actionperception sequence (Dietrich, 2015). In other words, a series of individual, uncreative steps can produce a final, creative configuration. This is different from the DM and SM in which a single step can be a creative act (Table 1).

\section{Conclusions}

Probing the cognitive and neural basis of creativity has been doggedly frustrated. There is a consensus view that further progress on this vexed problem must come from dividing the complex and multifaceted concept of creativity into different subtypes. However, attempts to do this have not returned subtypes of creativity that are valid, primarily because the proposals have been false category formations, still compound constructs, impossible to connect to mainstream knowledge, or in the case of divergent thinking, all three.

The present theoretical framework takes a new line of attack that avoids these problems by explicitly using the knowledge base of cognitive psychology and neuroscience to derive the different subtypes. This scheme yields valid subtypes of creativity that move the field significantly closer to conceptually sound empirical research and thus meaningful data.

A large amount of empirical data and theoretical concepts from three different levels of descriptions and domains was synthesized in the present framework. This synthesis also contains the results of recent work that probed, checked, and examined many first-time features of an earlier version of the framework. Based on established notions in (A) neuroanatomy, (B) processes, and (C) evolutionary algorithms,

Table 1 Types of creativity

\begin{tabular}{llll}
\hline \multicolumn{1}{c}{ Anatomy } & Processes & EAs \\
\hline DM & Explicit System & HCF Online & Higher Sightedness \\
& $\begin{array}{l}\text { PFC Activation } \\
\text { Hierarchical Network }\end{array}$ & Foresight and Agency & \\
SM & Explicit System & HCF Weakened & Lower Sightedness \\
& Mild TH & No Foresight and Agency & \\
& Even Network & Remote Associations & \\
FM & $\begin{array}{l}\text { Implicit System } \\
\text { Strong TH }\end{array}$ & HCF Offline & Blindness \\
& Implicit Network & Motor Effciency & \\
\hline
\end{tabular}

An oversimplified summary of the three types of creativity and their underlying mechanisms at three different levels of description

$D M$ deliberate mode, $S M$ spontaneous mode, $F M$ flow mode, $P F C$ prefrontal cortex, $H C F$ higher-cognitive functions, $T H$ transient hypofrontality creativity is parsed into three distinct types: a deliberate mode (DM), a spontaneous mode (SM), and a flow mode (FM). It is hoped that the present theoretical framework helps revitalize sound research on a mental faculty that defines humanity.

Acknowledgements I like to thank Patricia Montañés and Stephane Doncieux for helping in preparing this paper.

\section{References}

Abraham, A (2013). The promises and perils of the neuroscience of creativity. Frontiers in Human Neuroscience, 7, 1-9.

Abraham, A (2016). The imaginative mind. Human Brain Mapping, 37, 4197-4211.

Abraham, A (2018). The neuroscience of creativity. Cambridge University Press.

Allport, A., Styles, E. A. \& Hsieh, S. (1994). Shifting intentional set: Exploring the dynamic control of tasks. In: C. Umiltà \& M. Moscovitch (Eds.). Attention and performance 15. Attention and performance series, (pp. 421-452). Cambridge: MIT Press.

Baars, B. J. (1988). A cognitive theory of consciousness. Cambridge University Press.

Baird, B., Smallwood, J., Mrazek, M. D., Kam, J. W. Y., Franklin M. S., \& Schooler J. W. (2012). Inspired by distraction: mind wandering facilitates creative incubation. Psychological Science, 23, 11171122. https://doi.org/10.1177/0956797612446024

Bar, M. (2009). The proactive brain: Memory for prediction. Philosophical Transactions of the Royal Society B, 364, 12351243. https://doi.org/10.1098/rstb.2008.0310

Beaty, R. E., Benedek, M., Silvia, P. J., \& Schacter, D. L. (2016). Creative cognition and brain network dynamics. Trends In Cognitive Science, 20,87-95.

Beilock, S. L., \& Carr, T. H. (2005). When High-Powered People Fail. Working Memory and "Choking Under Pressure" in Math. Psychological Science, 16, 101-105.

Blackmore, S. (2005). Consciousness: A very short introduction. New York: Oxford University Press.

Boden, M. A. (1998). Creativity and artificial intelligence. Artificial Intelligence, 103, 347-356.

Bressler, S. L., \& Menon, V. (2010). Large-scale brain networks in cognition: emerging methods and principles. Trends in Cognitive Science, 14, 277-290. https://doi.org/10.1016/j.tics.2010.04.004

Carson, S. H., Peterson, J. B., \& Higgins, D. M. (2003). Decreased latent inhibition is associated with increased creative achievement in highfunctioning individuals. Journal of Personality and Social Psychology, 85, 499-506. https://doi.org/10.1037/0022-3514.85.3. 499

Chrysikou, E. G. (2018). The costs and benefits of cognitive control for creativity. In R. E. Jung \& O. Vartanian (Eds.), The Cambridge handbook of the neuroscience of creativity, (pp. 137-152). Cambridge University Press.

Csikszentmihalyi, M. (1996). Creativity. New York: Harper Perennial.

Cramond, B., \& Kim, K. H. (2009). The Torrance Tests of Creative Thinking. In B. Kerr (Ed.) Encyclopedia of giftedness, creativity, and talent, (pp. 900-902). Thousand Oaks: Sage.

Crick, F., \& Koch, C. (2003). A framework for consciousness. Nature Neuroscience, 6, 119-126.

Damasio, A. R. (1994). Descartes' Error: Emotion, Reason, and the Human Brain. New York: G. P. Putnam.

Dawkins, R. (1976). The selfish gene. Oxford: Oxford University Press. 
Dennett, D. C. (2004). Could there be a Darwinian account of human creativity? In A. Moya \& E. Font (Eds.), Evolution, from molecules to ecosystems. Oxford: Oxford University Press.

DeBono, E. (1968). New think: The use of lateral thinking in the generation of new ideas. New York: Basic Books.

Dehaene, S., \& Changeux, J.-P. (2011). Experimental and theoretical approaches to conscious processing. Neuron, 70, 200-227.

Dienes, Z., \& Perner, J. (1999). A theory of implicit and explicit knowledge. Behavioral and Brain Sciences, 5, 735-808.

Dietrich, A. (2003). Functional neuroanatomy of altered states of consciousness. The transient hypofrontality hypothesis. Consciousness and Cognition, 12, 231-256.

Dietrich, A. (2004a). The cognitive neuroscience of creativity. Psychonomic Bulletin \& Review, 11, 1011-1026.

Dietrich, A. (2004b). Neurocognitive mechanisms underlying the experience of flow. Consciousness and Cognition, 13, 746-761.

Dietrich, A. (2007a). Who is afraid of a cognitive neuroscience of creativity? Methods, 42, 22-27.

Dietrich, A. (2007b). Introduction to consciousness. London: Palgrave Macmillan.

Dietrich, A. (2015). How creativity happens in the brain. London: Palgrave Macmillan.

Dietrich, A., \& Audiffren, M. (2011). The reticular-activating hypofrontality (RAH) model of acute exercise. Neuroscience \& Biobehavioral Reviews, 35, 1305-1325.

Dietrich, A., \& Haider, H. (2015). Human creativity, evolutionary algorithms, and predictive representations: The mechanics of thought trials. Psychonomic Bulletin \& Review, 22, 897-915

Dietrich, A., \& Haider, H. (2017). A neurocognitive framework for human creative thought. Frontiers in Psychology: Cognitive Science, 7, 2078-2085.

Dietrich, A., \& Kanso, R. (2010). A review of EEG, ERP and neuroimaging studies of creativity and insight. Psychological Bulletin, 136, 822-848.

Dijksterhuis, A. (2006). On making the right Choice: The deliberationwithout-attention effect. Science, 311, 5763, 1005-1007.

Downing, K. L. (2009). Predictive models in the brain. Connection Science, 21, 39-74.

Dreisbach, G., \& Haider, H. (2009). How task representations guide attention: Further evidence for the shielding function of task sets. Journal of Experimental Psychology: Learning, Memory, and Cognition, 35, 477-486.

Fink, A., \& Benedek, M. (2014): EEG alpha power and creative ideation. Neuroscience Biobehavial Review, 44, 111-123.

Frith, C. D. (1992). The cognitive neuropsychology of schizophrenia. Hove: Lawrence Erlbaum.

Gigerenzer, G., \& Gaissmaier, W. (2011). Heuristic decision making. Annual Review of Psychology, 62, 451-482. https://doi.org/10. 1146/annurev-psych-120709-145346

Gould, S. J. (1979). Shades of Lamarck. Natural History, 88, 22-28.

Guilford, J. P. (1950). Creativity. American Psychologist, 5, 444- 454.

Guilford, J. P. (1967). The nature of human intelligence. New York: McGraw-Hill.

Grush, R. (2004). The emulation theory of representation: Motor control, imagery, and perception. Behavioral and Brain Sciences, 27, $377-$ 396.

Haider, H., \& Frensch, P. A. (2005). Empirical research on the generation and functional role of consciousness. Psychological Research, 69, 313-315.

Jamison, K. R. (1993). Touched with fire: Manic-depressive illness and the artistic temperament. New York: Free Press.

Jung, R. E., Mead, B. S., Carrasco, J., \& Flores, R. A. (2013). The structure of creative cognitionin the human brain. Frontiers in Human Neuroscience, 7, 330 .

Kerr, B., \& Gagliardi, C. (2003). Measuring creativity in research and practice. In S. J. Lopez \& C. R. Snyder (Eds.), Positive psychological assessment: A handbook of models and measures, (pp. 88-99). Washington D.C.: American Psychological Association.

Kronfeldner, M. E. (2007). Is cultural evolution Lamarckian? Biology \& Philosophy, 22, 493-512.

Kronfeldner, M. E. (2010). Darwinian "blind" hypothesis formation revisited. Synthese, 175, 193-218.

Kounios, J., \& Beeman, M. (2014). The cognitive neuroscience of insight. Annual Review of Psychology, 65, 71-93.

Lewontin, R. C. (1991). Biology as ideology. New York: Harper.

Maia, T. R., \& Cleeremans, A. (2005). Consciousness: converging insights from connectionist modeling and neuroscience. Trends in Cognitive Science, 9, 397-404.

Mayr, E. (1981). The growth of biological thought: Diversity, evolution and inheritance. Cambridge: Harvard University Press.

Martindale, C. (1995). Creativity and Connectionism. In S. M. Smith, T. B. Ward, \& R. A. Finke (Eds.), The creative cognition approach, (pp. 249-268). Cambridge: MIT Press.

Martindale, C. (1999). The biological basis of creativity. In R. J. Sternberg (Ed.), Handbook of creativity, (pp. 137-152). Cambridge: Cambridge University Press.

Mednick, S. (1962). The associative basis of the creative process. Psychological Review, 69, 220-232.

Miller, E. K., \& Cohen, J. D. (2001). An integrative theory of prefrontal cortex function. Annual Review of Neuroscience, 24, 167-202.

Monsell, S. (2003). Task switching. Trends on Cognitive Science, 7, 134140.

Moulton, S. T., \& Kosslyn, S. M. (2009). Imagining predictions: Mental imagery as mental emulation. Philosophical Transactions of the Royal Society B, 364, 1273-1280.

Norman, D. A., \& Shallice, T. (1986). Attention to action: Willed and automatic control of behavior. In R. J. Davidson, G. E. Schwartz, \& D. Shapiro (Eds.), Consciousness and self-regulation (pp. 1-18). New York: Plenum.

Nikolaidis, A., \& Barbey, A. K. (2018). Network dynamics theory of human intelligence. In R.E. Jung \& O. Vartanian (Eds.), The Cambridge handbook of the neuroscience of creativity, (pp. 137152). Cambridge University Press.

Pfenninger, K. H., \& Shubik, V. R. (2001). Insights into the foundation of creativity: a synthesis. In K. H. Pfenninger, \& V. R. Shubik (Eds.), The origins of creativity, (pp. 213-236). London: Oxford University Press.

Poldrack, R. A., \& Packard, M. G. (2003). Competition among multiple memory systems: Converging evidence from animal and human brain studies. Neuropsychologia, 41, 245-251.

Perruchet, P., \& Vinter, A. (2002). The self-organizing consciousness. Behavioral and Brain Sciences, 25, 297-388.

Raichle, M. E., MacLeod, A. M., Snyder, A. Z., Powers, W. J., Gusnard, D. A., \& Shulman, G. L. (2001). A default mode of brain function. Proceedings of the National Academy of Sciences, 98, 676-682. https://doi.org/10.1073/pnas.98.2.676

Ravizza, K. (1977). Peak performances in sports. Journal of Humanistic Psychology, 4, 35-40.

Reber, A. S. (1993). Implicit learning and tacit knowledge. Oxford: Oxford University Press.

Richerson, P. J. \& Boyd, R. (2005). Not by genes alone: How culture transformed human evolution. Chicago: University of Chicago Press.

Runco, M. (2004). Creativity. Annual Review of Psychololgy, 55, 657-87.

Sawyer, K. (2011). The cognitive neuroscience of creativity: A critical review. Creativity Research Journal, 23, 137-154.

Schooler, J. W., \& Melcher J. (1995). The ineffability of insight. In S. M. Smith, T. B. Ward, \& R. A. Finke (Eds.), The creative cognition approach, (pp. 97-133). Cambridge: MIT Press.

Simonton, D. K. (1975). Creativity, task complexity, and intuitive versus analytical problem solving. Psychological Reports, 37, 351-354. 
Simonton, D. K. (2015). On praising convergent thinking: Creativity as blind variation and selective retention. Research Creativity Journal, 27, 262-270.

Simonton, D. K. (2013). Creative thought as blind variation and selective retention: Why creativity is inversely related to sightedness. Journal of Theoretical and Philosophical Psychology, 33, 253-266.

Singer, J. L. (1975). Navigating the stream of consciousness: Research in daydreaming and related inner experiences. American Psychologist, $30,727-738$

Smith, E. A. (2013). Agency and adaptation: New directions in evolutionary anthrophology. Annual Review of Anthropology, 41, 103120

Smith, S. M., Ward, T. B., \& Finke, R. A. (1995). The creative cognition approach. Cambridge: MIT Press.

Torrance, E. P. (1974). Torrance test of creative thinking. Lexington: Personal Press.

Uttall, W. R. (2001). The new phrenology. Cambridge: MIT Press.

Wagner, U., Gais, S., Haider, H., Verleger, R., \& Born J. (2004). Sleep inspires insight. Nature, 427, 352-55.
Ward, T. B., Smith, S. M., \& Finke, R. A. (1999). Creative cognition. In R. J. Sternberg (Ed.), Handbook of creativity, (pp. 189-212). Cambridge University Press.

Weisberg, R. W. (2013). On the demystification of insight: A critique of neuroimaging studies of insight. Creativity Research Journal, $25,1-14$.

Whittlesea, B. A. (2004). The perception of integrality: Remembering through the validation of expectation. Journal of Experimental Psychology: Learning, Memory, and Cognition, 30, 891-908.

Wolpert, D. M., Ghahramani, Z., \& Jordan, M. I. (1995). An internal model for sensorimotor integration. Science, 269, 1880-1882.

Wolpert, D. M., Doya, K., \& Kawato M. (2003). A unifying computational framework for motor control and social interaction. Philosophical Transactions of the Royal Society Series B: 358, 593-602. https://doi.org/10.1098/rstb.2002.1238

Yoruk, S., \& Runco, M. A. (2014). The neuroscience of divergent thinking. Activitas Nervosa Superior, 56, 1-16.

Zabelina, D. (2018). Attention and creativity. In R. E. Jung \& O. Vartanian (Eds.), The Cambridge handbook of the neuroscience of creativity, (pp. 137-152). Cambridge : Cambridge University Press. 